\title{
A proposed solution for airborne delays: linear holding
}

\author{
O. Sahin
}

ozlemsahin@eskisehir.edu.tr

Faculty of Aeronautics and Astronautics

Department of Air Traffic Control

Eskisehir Technical University

Turkey

\section{ABSTRACT}

This study is aimed at establishing a linear holding $(\mathrm{LH})$ procedure instead of a conventional air holding stack to minimize the effects of airborne delays in terms of air traffic management and fuel consumption. This paper uses both actual flight data and the Base of Aircraft Database (BADA) model to obtain fuel consumption for level flight and descent segments, separately.

The total fuel savings obtained by using actual flight data (16\%) and the BADA model $(10 \%)$ indicate that the $\mathrm{LH}$ is found to be more advantageous compared to a conventional holding procedure. Furthermore, the recommended LH procedure could be a promising solution for keeping aircraft in a narrow area that could be considered to be an effective method for airspace usage.

Keywords: Air Traffic; Linear Holding; Aircraft Fuel Consumption; Airborne Delay

\section{NOMENCLATURE}

ACC Area control centre

ATC Air traffic control

AFP Airspace flow program

AIP Aeronautical Information Publication

AMSL Above mean sea level

ATM Air traffic management

BADA Base of Aircraft Database

BEST Beginning to End for Simulation and Training

CDA Continuous Descent Approach

CTR Control zone

EAT Expected approach time

EEC EUROCONTROL Experimental Centre

FB' Fuel burn

FCFS First-come-first-served 


$\begin{array}{ll}\text { FF } & \text { Fuel flow } \\ \text { FPA } & \text { Flight path angle } \\ \text { GDP } & \text { Ground delay program } \\ \text { GNSS } & \text { Global navigation satellite system } \\ \text { LH } & \text { Linear holding } \\ \text { LTAR } & \text { Sivas Nuri Demirag Airport } \\ \text { PM } & \text { Point merge } \\ \text { RNAV } & \text { Area navigation } \\ \text { RTS } & \text { Real-time simulation } \\ \text { SR' } & \text { Specific range } \\ \text { STAR } & \text { Standard arrival route } \\ \text { TAS } & \text { True air speed } \\ \text { TLA } & \text { Throttle lever angle } \\ \text { TMA } & \text { Terminal control area }\end{array}$

\subsection{INTRODUCTION}

Terminal control areas (TMAs) could be considered as complex and congested airspace since they are having to accept more flights each day, so up and down link disruptions inevitably occur. However, the growth in air traffic demand, especially during peak times, is approaching TMA maximum capacity, where there is almost no possibility of creating new infrastructure. Therefore, delays generally occur because of an imbalance between high traffic demand and insufficient capacity, as well as other factors such as air traffic management, weather, airports, passengers, airlines and so on ${ }^{(1)}$.

Attempts including new concepts, procedures and tools have been undertaken to improve TMA efficiency: for instance, a reduction in delays; modification of air traffic handling methods based on modern avionic capabilities so as to reduce additional separation and to sequence traffic flow; modification of arrival/departure procedures with better air routes in order to develop flexibility and to provide more economical flights; and performing re-sectorization such as geographical and functional methods to enable faster and safer traffic flow ${ }^{(2,3)}$.

Delays are unavoidable situations, especially in high density TMAs, and therefore it is important to achieve safer and more economical flight operations. Delays could occur either on the ground or in the air ${ }^{(4)}$. Rerouting, air holding stacks ${ }^{(5)}$ or path stretching ${ }^{(2)}$ could be considered as forms of airborne delays in TMAs. Of these, air holding stacks are a conventional method and the most widely used, especially in airspace in which procedural control service is given. Through the air navigation service provider, a holding stack is constructed based on entry and holding procedures and is published in Aeronautical Information Publications (AIPs). If these procedures are not published or known to a flight crew, a controller should give necessary acknowledgment such as which navigation aid to be used, the inbound track, radial or bearing, direction of turn in the holding pattern, as well as times for the outbound leg or distances. Besides the holding instructions, the controller is responsible for giving the expected approach time or onward clearance time ${ }^{(6)}$.

Levels at a holding fix should be assigned in order to ensure that each aircraft approaches in the correct priority. The controller normally gives the lowest level to the first aircraft, which arrives over a holding fix. However, in the case of two or more aircraft and the first aircraft keeps a higher level without having any longitudinal separation, the controller is unable to give the lowest level to the first aircraft due to a lack of required separation. The aircraft should enter the holding stack maintaining their levels. In this situation, the aircraft at the lowest 
level leaves the holding first. Vertical separation is required between the aircraft holding and other aircraft, except when lateral separation exists. Any speed control instruction should not be given to the aircraft entering or established in a holding stack $^{(6)}$.

In the case of extended delays, the controller could give a speed reduction instruction in the en route phase in order to absorb any delay in TMAs. In fact, speed control strategies for air traffic management (ATM) are generally used in order to resolve aircraft conflicts and to achieve traffic synchronization. However, by reducing aircraft nominal speed in the cruise, aiming to extend flight duration, aircraft are delayed. Numerous research studies have focused on many different solutions to airborne delays. Gunther and Fricke ${ }^{(7)}$ reveal potential flight efficiency improvements by reducing cruise speed and aim to prevent entry into airborne holding patterns when aircraft arrive at congested TMAs.

Air services Australia developed an ATM long-range optimal flow tool for Sydney Airport, which is aimed at preventing aircraft arriving before the airport is open and entering unnecessary holding patterns by reducing flight speed ${ }^{(4)}$. Delgado and Prats ${ }^{(8)}$ focus on the wind and assess the effects of wind on cruise speed reduction methods applied to airborne delays while maintaining fuel burn.

In addition to airborne delays, aircraft are delayed on the ground prior to their departure from airports of origin. For this purpose, several studies focus on the improvement of Ground Delay Programs (GDPs) or Airspace Flow Programs (AFPs) ${ }^{(4,8)}$. As long as a delay is unavoidable, the thinking is that delaying on the ground at airports of origin, rather than delaying aircraft in the air, reduces fuel consumption and environmental impact.

Moreover, other studies attempt to harmonize ground delay strategies by including cruising speed adjustments ${ }^{(9-13)}$, scheduling and sequencing traffic flows with different optimization models ${ }^{(14-16)}$. Furthermore, Akturk et al. ${ }^{(17)}$ focus on fuel consumption and $\mathrm{CO}_{2}$ emissions costs incorporating cruise speed control by proposing a novel rescheduling optimization model.

In addition to these delay methods, nowadays, area navigation (RNAV) arrival routes, that use linear holding (LH) procedures, such as 'Point Merge (PM)', are preferable to vertical conventional holding stacks. LH could be designed into an RNAV standard arrival route (STAR). LH is characterized by a single point and predefined variable legs. It is designed to delay, sequence and integrate arrival aircraft without heading instructions. Moreover, the need for conventional holding stacks could be reduced or replaced entirely using LH procedures ${ }^{(18)}$.

In the case of overloading the capacity of predefined legs, holding stacks could be established before entering the arrival procedure. In the LH procedure, the controller allows aircraft to fly and complete the longest linear hold route, which is defined as a series of arcs. At the appropriate time while creating the spacing, each aircraft is given a 'direct to' instruction to the merge point. After leaving the legs, the spacing between aircraft is provided by speed control $^{(18-20)}$.

PM, a type of LH, is already in operation at certain airports: Oslo (2011), Dublin (2012), three Norwegian regional airports (2014), Seoul (2012), Paris ACC (2013), Kuala Lumpur (2014), Lagos (2014), Canary Islands (2014), Hannover (2014), Leipzig (2015), London Terminal Control Centre for London City and Biggin Hill $(2016)^{(21,22)}$. Moreover, for Turkey's third airport recently built in Istanbul (2018), the PM will be in operation on 29 October 2018.

Most previous studies related to the PM system have focused on providing a reduction in the total number of controller instructions, frequency occupancy time as well as fuel consumption and environmental impact by enabling Continuous Descent Approaches $(\mathrm{CDA})^{(19-30)}$, which allow aircraft to descend continuously by employing minimum engine thrust, ideally in low drag configuration, prior to the final approach fix/final approach point ${ }^{(31)}$. 
LH has been applied in Dublin since 2012. Rather than using conventional holding stacks, an arriving aircraft is delayed onto defined equidistant arcs or tracks, from which they can make a continuous descent to a runway. It can be seen that both flight distance flown, and fuel burn are reduced ${ }^{(32)}$. In the literature, it is noted that studies related to a comparison between conventional holding and LH procedures are limited and only focus on UK airspace.

This study is focused on establishing a LH method for Sivas TMA instead of a conventional air holding stack. The LH procedure is designed and proposed for every runway. LH and conventional holding are compared using real flight data. The results are analysed using the Real-Time Simulation (RTS) method in terms of air traffic management. Furthermore, fuel consumption results based on real flight data and the BADA (Base of Aircraft Database) model are obtained.

\subsection{PROBLEM DESCRIPTION}

There are numerous constraints and assumptions in the airspace design, such as terrain limitations, special-use areas (prohibited, restricted, dangerous, etc.), navigation infrastructure, adjacent aerodromes/sectors, traffic mix and the level of air traffic control services (procedural, radar). These local constraints determine the arrival/departure and holding procedures for each airspace, individually. Furthermore, modifications in airspace design and management could create rearrangement and additional requirements. Recently, Sivas Nuri Demirag Airport (LTAR) changed their airspace design in order to meet requirements to provide safer and more effective aerodrome and procedural control services. Before the regulations are followed in the airspace, an air traffic control service is provided within a 15-nm radius control zone (CTR) up to 13,000 ft AMSL; however, this meant air traffic controllers were to achieve separation between aircraft. In order to meet the requirements, some of the changes, such as designing RNAV arrival/departure routes, RNAV GNSS approach/departure procedures and designing a TMA with a $25-\mathrm{nm}$ radius have been undertaken. However, switching from CTR to TMA is required to implement airspace re-design. Modification in the dimensions of airspace and establishing new holding stacks have created a number of problems with adjacent airports/TMAs, such as at Tokat, Malatya and Erzurum.

In Fig. 1, four holding stacks are indicated and which number of them appear sufficient; however, it might not be possible to use them simultaneously because of overlapping buffer areas. Moreover, the holding stacks might also affect other adjacent sectors due to their locations.

LTAR has only one runway with a $01 / 19$ configuration $^{(33)}$. The TMA has five entry points, as indicated in Fig. 1. Once an arriving aircraft enters the TMA, it should proceed to the runway. However, before aircraft are guided through their landing procedure, they could be delayed by flying in circles in specific areas called holding stacks.

When entering a holding stack, an aircraft should apply inbound and outbound turns at 180 degrees at a specified level and for a specified time period ${ }^{(34)}$. Aircraft should remain in a holding area, which is defined in terms of aircraft speed, wind effect, timing errors, holding fix characteristics and so on. In addition, a 5-nm buffer zone is added in the vicinity of the holding area in order to determine the holding level according to obstacles ${ }^{(35)}$.

In the LTAR TMA, four conventional holding stacks are available; two of these (GOPOX and EKROR) are located to the north, while the others (ATNAX and OKOTU) are located to the south at $10,000 \mathrm{ft}$ and at $9,000 \mathrm{ft}$, respectively. 


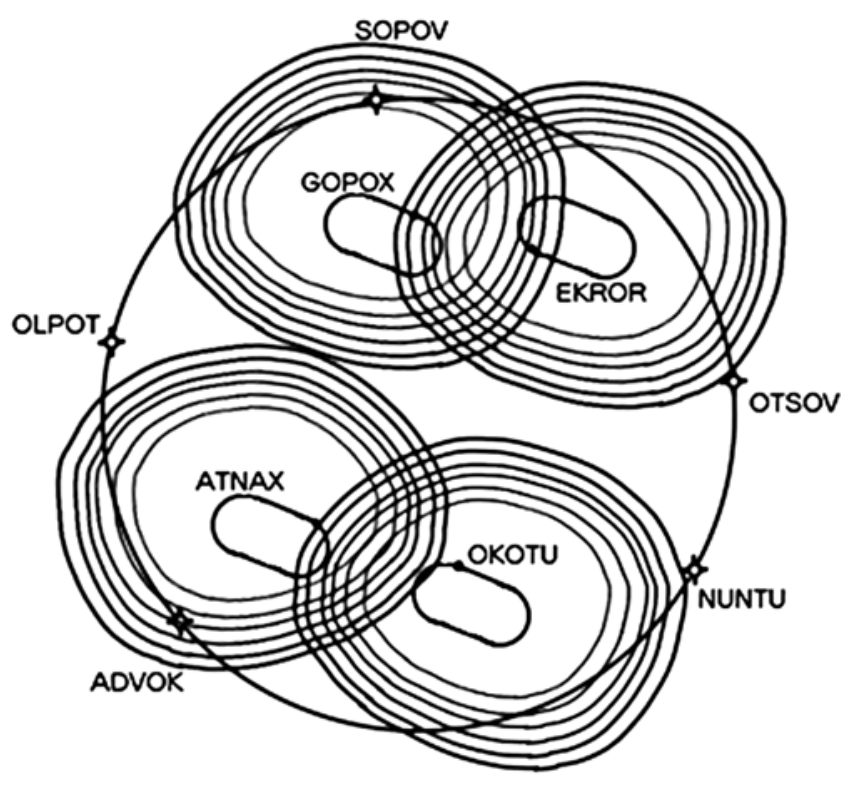

Figure 1. The illustration of the boundary of LTAR TMA with entry points and conventional holding stacks.

In the LTAR TMA, the numbers of holding stacks appear to be sufficient. However, it is not possible to use them simultaneously due to an overlapping of their buffer areas. Moreover, using the GOPOX and Tokat holding stacks is necessary for coordination between air traffic controllers in adjacent sectors. Furthermore, aircraft use the SOPOV and/or OTSOV points for entry/exit points for the TMA, and furthermore, aircraft that are delayed over the EKROR point are affected by the boundary of Merzifon airspace. Moreover, the boundary of Malatya, which violates the LTAR TMA, impacts aircraft holding over an OKOTU point.

The arrival/departure traffic flows are generally from a westerly direction, and therefore, ATNAX and GOPOX are the most widely used holding stacks, providing separation between the arrival and departure aircraft. However, while applying holding stacks, the number of holds to be flown or where the controller will vector the aircraft are not known. Even though adequate separation between planes is achieved, the aircraft should complete the entire holding procedure in approximately four minutes (at and below 14,000 ft), corresponding to a 20 -nm flight distance. Furthermore, for the purpose of saving fuel and in order not to be affected by icing on cold days, arrival traffic performing holding at higher levels is preferable. In the case of holding at a higher level, wider holding areas that affect adjacent airports occur.

In this paper, the goal is to develop an airspace redesign in order to delay aircraft, if necessary, on predefined legs at higher levels and to provide more economical aircraft operations. For this goal, a LH is proposed and compared with the available conventional holding procedure in terms of air traffic management and fuel consumption using real flight data.

\subsection{METHODOLOGY}

It should be noted that the number and direction of predefined routes could be changed according to requirements and the complexity of traffic operations. In the case of there being more than one leg, which are vertically separated, then the separation between the aircraft flying 
along the route could therefore be guaranteed. The purpose of the LH is to delay aircraft on predefined routes, defined as a series of arcs and dedicated to path stretching. On the predefined legs, aircraft keep as high a level as possible rather than following conventional arrival routes. After entering the $\mathrm{LH}$, an aircraft flies at a constant speed along the leg. At the appropriate time, when achieving separation, each aircraft is given a 'direct to' instruction to the merge point. After leaving the legs, the spacing between the aircraft is provided by speed control $^{(19,36)}$.

LH based on PM was first developed by the EUROCONTROL Experimental Centre (EEC) in 2006. LH could be designed into an RNAV STAR, which is characterized by a single merge point and predefined legs ${ }^{(36-38)}$. These legs provide sequencing, and they play a critical role in absorbing necessary delays by stretching paths ${ }^{(39)}$.

According to a 2017 statistical analysis of an air navigation service provider, 4,173 traffic movements are declared annually in $\mathrm{LTAR}^{(40)}$. It is not a busy TMA and provides a procedural approach control service, so there is no surveillance system. It should be noted that efficient and effective airspace design is so significant such that adjacent airports are where the air traffic control service is not using a surveillance system in order to provide aircraft operations in safety and to increase controller awareness.

A single point and only one predefined leg define the LH for each runway in order to integrate traffic flows from different directions. The Sivas TMA entry points remain in the same locations. For runway 19, the arrival traffic flow from the northwest and northeast use the OLPOT and OTSOV entry points, respectively. The ADVOK and NUNTU entry points, located to the southwest and southeast, are used by arrival traffic flows for runway 01. Air holding stacks are located $16 \mathrm{~nm}$ away from runway 01 and runway 19; in other words, all are equidistant from the thresholds. Therefore, in this paper, one air holding stack, GOPOX, which is located to the northwest and most often used, is chosen for comparison with the proposed LH.

In LTAR TMA, RNAV (GNSS) an instrument approach procedure is stated as a baseline. In order to provide separation between arriving-arriving or arriving-departing traffic, aircraft are held in air holding stacks at a specified altitude and flight speed.

The GOPOX holding stack is used for traffic from the OLPOT entry point. Therefore, while preparing a traffic scenario, arrival traffic from the OLPOT is taken into consideration. In the proposed LH procedure, the traffic flows from the OLPOT join the LH on the IAWP. Aircraft should apply a speed reduction to $220 \mathrm{Kn}$ before entering the LH procedure. The distance of the predefined legs is determined as $30 \mathrm{~nm}$. Due to a lack of radar capabilities in the LTAR, the predefined waypoints (X, M, T, N and S) are defined at one-minute intervals in order to check positioning and to provide separation. While determining the level limitation of the predefined leg, a descent gradient $(6.5 \%)$ is taken into consideration, and the leg is defined at a maximum 14,000 ft. The distance between the merge point and predefined leg is $16 \mathrm{~nm}$. As referred to in Fig. 2, there are two predefined legs, the inner and outer legs, in order to merge aircraft from different directions. If needed, the number of legs may be increased or decreased. The proposed LH model is presented in Fig. 2.

An air traffic control (ATC) simulation system with 'Beginning to End for Simulation and Training' (BEST) software is used for preparing and testing the proposed LH procedure. The scenario that was created by using ATC simulation system includes two arriving and one departing aircraft. The types of aircraft are Boeing 737-800, and the aircraft performance data is derived from the BADA.

Two arriving on the same track without any longitudinal separation are entering TMA from the OLPOT. The preceding and following aircraft are at 14,000 ft and 12,000 ft, respectively. 


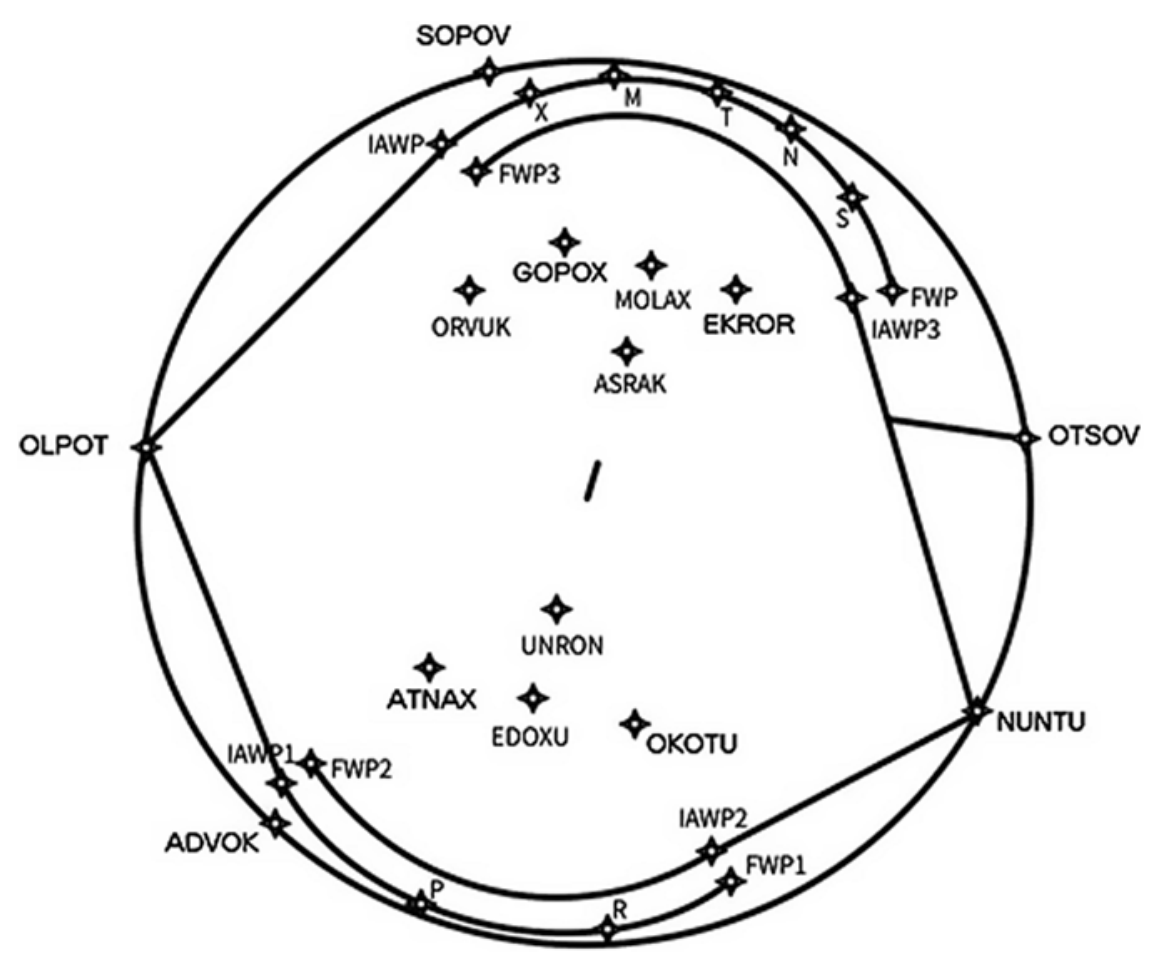

Figure 2. The proposed LH procedure and screenshot from BEST.

The RTS method is used, and the prepared scenario is tested five times by applying baseline and LH procedures, separately. Therefore, five controllers are assigned to test the scenario by using the procedural controller and pseudo-pilot position simultaneously.

While determining the duration of simulation, the time period between entry point and touchdown is taken into consideration. The scenario takes an average of 16 minutes from OLPOT to touchdown.

For the baseline procedure, it is considered that delaying aircraft performs one hold at 230 Kn IAS. One hold corresponds to approximately four minutes, or $20 \mathrm{~nm}$. Moreover, for the entire arrival segment, the flight distance is measured from flight paths published in AIP.

Furthermore, procedural separation minimums, the lateral global navigation satellite system (GNSS) based on separation and longitudinal separation of 15 degrees, $15 \mathrm{~nm}$ and $10 \mathrm{~nm}$, are applied, respectively. Arriving aircraft are transferred to Sivas TMA at 5-minute intervals at 22,000 ft in accordance with an arrangement with the Ankara area control centre (ACC). After the simulation, the exercise is recorded, and an evaluation of the differences between a holding stack and $\mathrm{LH}$ is conducted.

The LH procedure was benchmarked against the baseline procedure using actual flight data for a similar type of aircraft ${ }^{(41)}$. Uniquely, 2,693 actual flights were analysed focusing on descent flight between 22,000 ft and 10,000 ft. Two fuel consumption models, one for level flight at different altitudes and the other for the descent segment at different altitudes and the flight path angle (FPAs), are developed.

Regardless of flight phase, it is difficult to obtain a robust model for fuel flow (FF) since there are numerous factors that cause changes in the FF of an engine. For the cruise phase, 
(a)

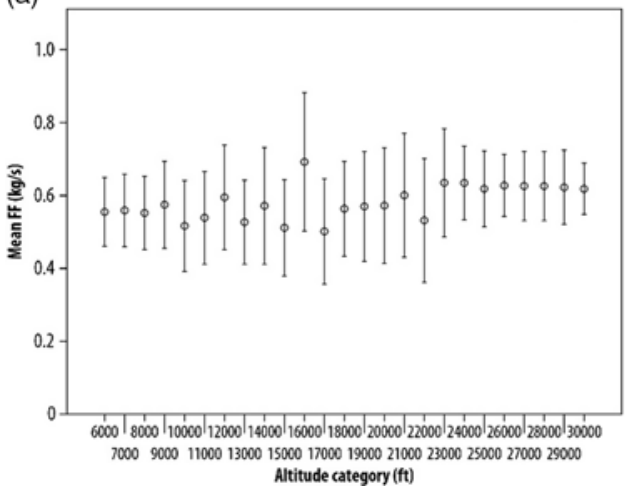

(b)

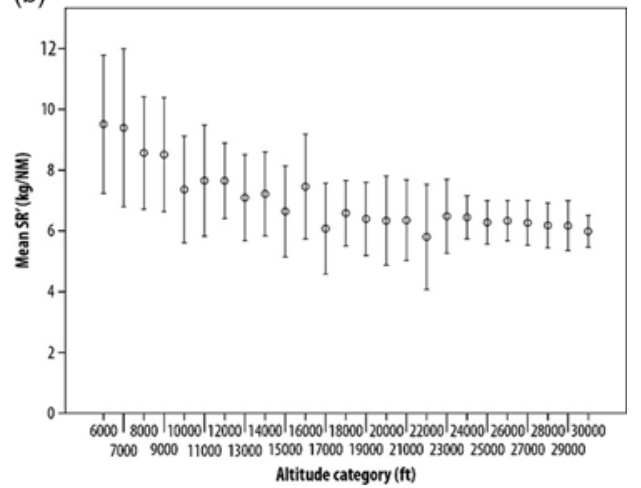

Figure 3. The variation of (a) FF and (b) SR' with altitude during level flight.

these factors may include cruise altitude and speed, weather conditions, aircraft weight and other engine loads (e.g. engine accessories, pneumatic air system). For phases such as climb and descent, FPA or vertical speed must also be introduced as another important parameter. Therefore, in these phases, explaining changes in FF becomes more challenging.

The effect of altitude on level flight FF for the dataset is illustrated in Fig. 3a. Since none of the other confounders are isolated, it is not possible to ascertain a clear effect of altitude on FF. However, while it is highly variable for the majority of the altitude categories, the mean FF appears to be virtually constant above $24,000 \mathrm{ft}$.

While analysing the flight data, it can be seen that level flights are performed for slowing down or maintaining specific flight speed. Therefore, thrust should be kept to a minimum, or a higher thrust is required that results in higher fuel burn. In this study, there is no distinction for flight speed, whether constant or variable. With the aim of reducing the effect of flight speed variations on $\mathrm{FF}$ and understanding if FF variation is based on altitude in level flight, or another parameter, the specific range (SR') can be used as follows ${ }^{(42)}$ :

$$
S R^{\prime}=\frac{T A S}{F F}
$$

where TAS refers to true airspeed (knot), and FF is fuel flow $(\mathrm{kg} / \mathrm{h})$. In this study, in order to note differences, the inverse version (SR') of Equation (1) is used. The variation on mean SR' (based on TAS) in terms of altitude is presented in Fig. 3b. The first observation made is that the higher the level flight altitude the lower is the SR'. In addition, it is also noted that the effect of altitude on SR' appears to be stronger for a change in lower altitude categories. The effects become weaker for higher altitudes. Recall that FF also shows a similar pattern for higher altitude levels. This pattern of change can be attributed to a speed change with altitude, which is comparatively lower for higher altitudes.

During the descent segment, height, FPA, flight speed and throttle lever angle (TLA), which define the thrust of the engine, are significant variables for FF variation. To decrease the variation on FF, descents only at a constant TLA ( $\pm 1 \%$ change accepted $)$ are identified and derived from the flight data records.

As shown in Fig. 4a, while the effect of FPA on FF is weak, a slight decrease in FF with increasing FPA can be noted. However, the FF of descents at higher altitude is clearly seen to 

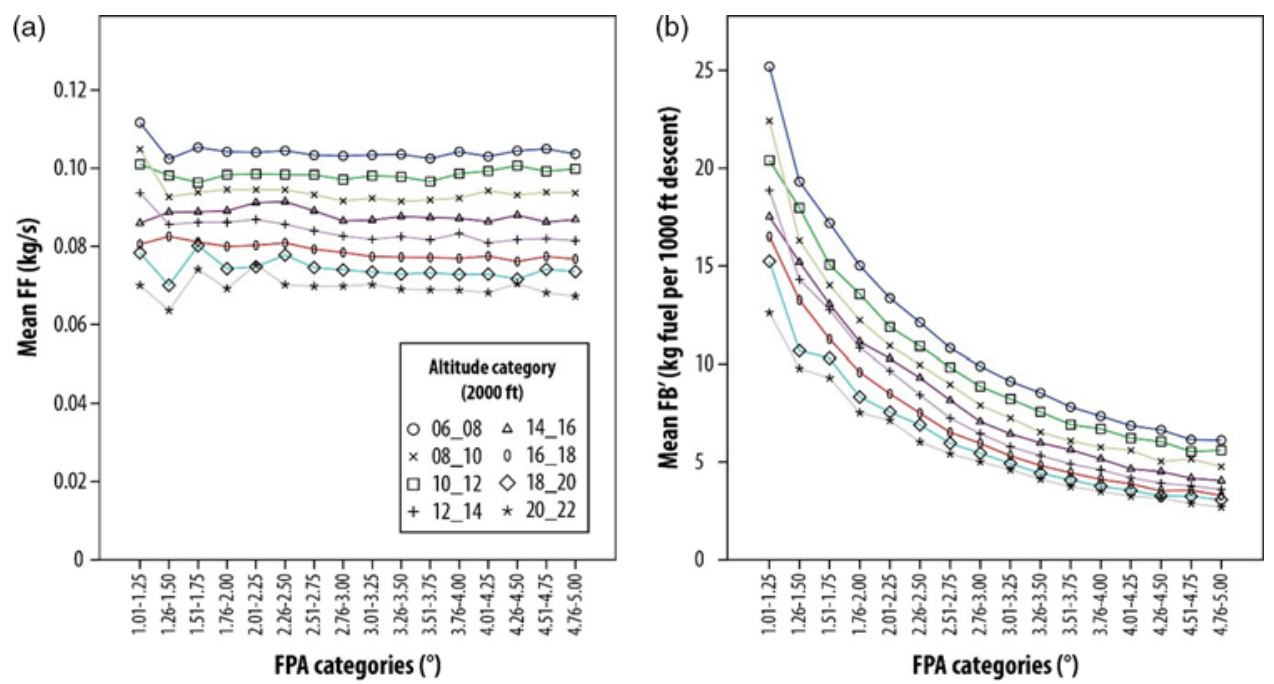

Figure 4. The variation of (a) FF and (b) FB' with FPA categories during descent.

be lower. Since, in this study, the effect of FPA on fuel consumption is important and the FF cannot be used alone to explain this relationship, a new parameter is developed that is defined as fuel burn per 1,000 ft (FB'). This parameter is used to explain how much fuel is burned during descent to $1,000 \mathrm{ft}$ at different altitude categories.

The relationship between FPA and FB' is illustrated in Fig. 4b. To reduce the number of series, it should be noted that the FB' values are plotted for 2,000 ft of altitude categories.

The effects of altitude with a constant FPA (considered as 3.01-3.25 degrees) on FB' are analysed. For instance, the FB' is $8.2 \mathrm{~kg}$ between altitudes of $8,000-10,000 \mathrm{ft}$, whereas for an altitude of $10,000-12,000 \mathrm{ft}$, the FB' is found to be $7.3 \mathrm{~kg}$ with a reduction of $11 \%$. As a result, with a constant FPA, a reduction of up to $10 \%$ is observed up to $14,000 \mathrm{ft}$. Between the altitudes of $14,000-22,000 \mathrm{ft}$, the FB' reduction is observed to be less than $10 \%$.

\subsection{RESULTS AND DISCUSSION}

In TMAs, where procedural air traffic control service is given, air holding stacks are widely used as an airborne delay method if needed. Although it is a commonly used method, it is uncertain how many holds will be flown. Performing more holds results in more fuel burn. However, in the recommended procedure, after a preceding aircraft reports passing a specific point, MOLAX, a following aircraft does not need to further complete a holding procedure. After checking position, a controller could give an instruction using predefined waypoints as geographical references.

In the Sivas TMA, the holding stacks area is very close to the final approach waypoint. For this reason, aircraft should descend in a specified altitude in a holding stack. In the case of entering hold at a higher level, the aircraft must enter a holding stack one more time to lose altitude. By implementing the recommended procedure, the aircraft could perform a hold at a higher preferable altitude on a predefined leg and descend with a $6.5 \%$ descent gradient in order to apply landing procedures. 


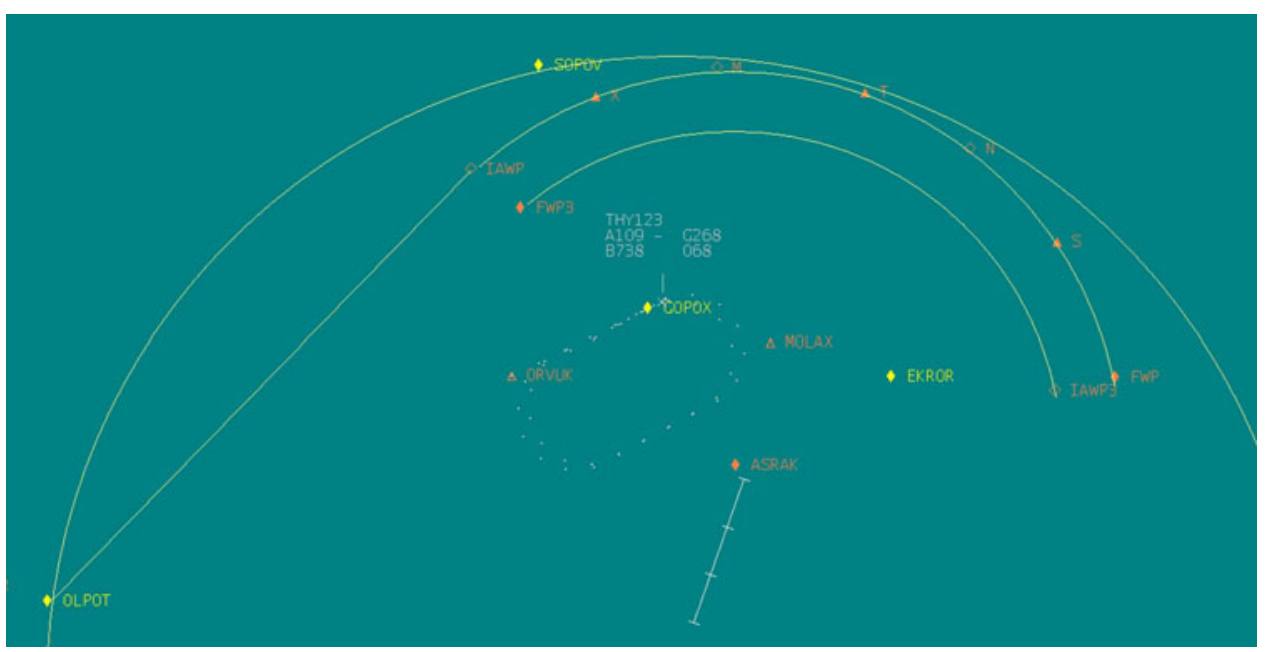

Figure 5. Conventional holding stack in RTS.

Furthermore, the recommended LH could merge and integrate aircraft from different directions allowing reciprocal aircraft to join and hold on predefined legs at higher altitudes. The number of legs may be increased or decreased as necessary. In order to perform holding for reciprocal aircraft simultaneously, two opposite predefined legs at different altitudes are established (Fig. 5). However, in the study area, four holding stacks are used for aircraft from different directions. This appears sufficient; however, it is not possible to use them simultaneously because of overlapping buffer areas. For instance, GOPOX and EKROR; ATNAX and OKOTU could not be used simultaneously.

In conventional holding procedures, the aircraft are ordered according to altitudes and entry time of holding. However, while leaving the holding stacks, an aircraft at the lowest altitude leaves the holding first. It is not a balanced rule in air traffic management. A 'first-come-firstserved' (FCFS) principle is accepted as a fair method of sequencing aircraft at most airports. In FCFS policy, aircraft are sequenced based on expected approach time (EAT) to a runway or a given point ${ }^{(43,44)}$. The results of the RTS analyses present that FCFS could be provided by implementing the LH procedure. Moreover, in the RTS scenario, in the case of two sametrack traffic reaching the GOPOX at 3-minute intervals, the second traffic is given an EAT 6 minutes later than the first aircraft. However, with conventional holding, implementing the $\mathrm{LH}$ procedure, the second traffic flies on a predefined leg until the first traffic passes a common point, called MOLAX (Fig. 6). The longer time holds of 6 minutes are not required. In Fig. 6, it appears that the first aircraft reached the common point; therefore, the following aircraft does not need to join and hold on the sequencing leg. and the controller could give direct instruction to a common point without delay.

The obstacle clearance area of holding consists of a protection area and buffer areas. In the case of overlapping obstacle clearance areas, the adjacent holding stacks cannot be used simultaneously. In other words, aircraft cannot be held at the same time and at the same altitude.

The radius depends on the square of TAS. As is known, air speed increases at higher altitudes. Therefore, the holdings at higher levels occupy a larger area due to buffer zones; therefore, traffic flows of adjacent sectors are affected. Moreover, in the case of remaining at 


\section{Table 1 \\ Flight distance, time, FPA and FB' for a conventional holding}

$\begin{array}{lccccr}\text { Altitude (ft) } & \text { Waypoint } & \begin{array}{r}\text { Flight dist. } \\ \text { (NM) }\end{array} & \begin{array}{r}\text { Flight time } \\ \text { (minute) }\end{array} & \text { FPA }\left(^{\circ}\right) & \begin{array}{r}\text { FB' } \\ (\mathbf{k g})\end{array} \\ 22,000-11,000 & \text { OLPOT-GOPOX } & 26 & 5 & 3.98 & 43.4 \\ 11,000 & \text { GOPOX (hold) } & 20 & 4 & 3.98 & 155.2 \\ 11,000-10,000 & \text { GOPOX-MOLAX } & 5 & 1 & 1.89 & 12.7 \\ & \text { Total } & \mathbf{5 1} & \mathbf{1 0} & & \mathbf{2 1 1 . 3}\end{array}$

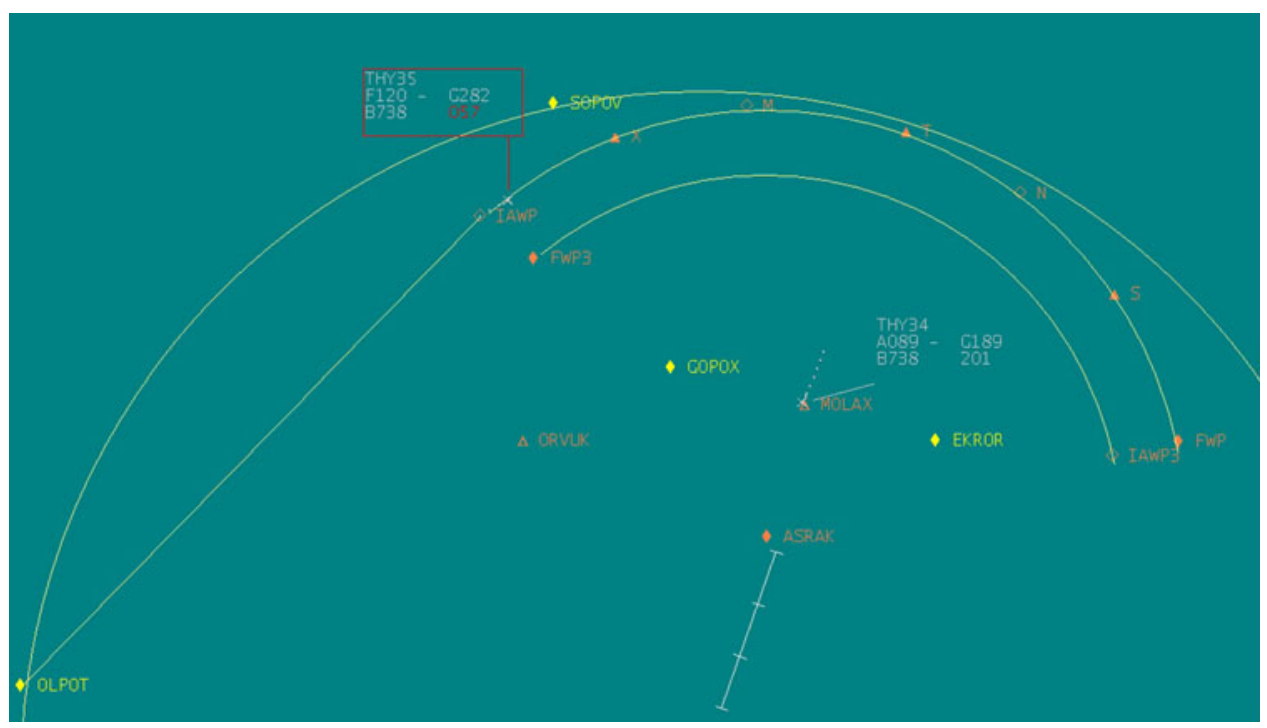

Figure 6. Applying FCFS in RTS.

higher altitudes, due to the location of GOPOX, aircraft are unable to lose altitude for landing procedures and must perform a 'going around' procedure. The recommended LH procedure could be one solution for keeping aircraft in a narrow area without any buffer zones. In addition, LH allows aircraft to fly at the end of the leg. This could be considered an efficient and effective method for airspace design and management.

In TMAs, where procedural air traffic control service is provided, due to a lack of radar capabilities, the responsibilities of controllers are increased. From an air traffic controller's perspective, the proposed LH procedure allows visualization of traffic flows easily, by having the predefined legs and waypoints to be followed. Therefore, an LH enables an increment in the situational awareness of controllers (and of pilots) to notify accurate positions of aircraft. Moreover, in RTS, controller feedback related to managing traffic and providing separation is in favour of the recommended LH procedure.

For a conventional holding procedure, flight distance and FB' is found to be $51 \mathrm{~nm}$ and $211 \mathrm{~kg}$, respectively (Table 1). In the proposed LH procedure, while the results of the flight distance are observed to be approximately the same at $50 \mathrm{~nm}$, the fuel burn is found to be 


\section{Table 2}

Flight distance, time, FPA and FB' for a proposed LH

$\begin{array}{lccccc}\text { Altitude (ft) } & \text { Waypoint } & \begin{array}{c}\text { Flight dist. } \\ \text { (NM) }\end{array} & \begin{array}{c}\text { Flight time } \\ \text { (minute) }\end{array} & \text { FPA }\left(^{\circ}\right) & \begin{array}{r}\text { FB' } \\ \text { (kg) }\end{array} \\ 22,000-14,000 & \text { OLPOT-IAWP } & 24 & 5 & 3.14 & 41.0 \\ 14,000 & \text { IAWP-T (seq. leg) } & 15 & 3 & 3.14 & 111.9 \\ 14,000-10,000 & \text { T-MOLAX } & 11 & 2 & 3.43 & 24.3 \\ & \text { Total } & \mathbf{5 0} & \mathbf{1 0} & & \mathbf{1 7 7 . 2}\end{array}$

$16 \%$ lower $(177 \mathrm{~kg})$ compared to the conventional procedure (Table 2). Moreover, for both conventional and $\mathrm{LH}$ procedures, the flight time is found to be 10 minutes.

Interestingly, if the level flights at descent had not been considered, the proposed LH procedure would result in a fuel penalty of $9 \mathrm{~kg}$. As shown in Fig. 4b, the reason for this is that a shallower FPA is executed for the LH procedure, and a steeper FPA yields a lower fuel consumption. Moreover, a $0.25 \%$ increase in FPA causes a reduction in FB'. For example, descending with an FPA of 1.26-1.50 is compared to an FPA 1.51-1.75 in terms of the FB' for altitudes of $6000 \mathrm{ft}-8000 \mathrm{ft}$. In the case of descending with an FPA of 1.51-1.75, a reduction of $10.9 \%$ in the $\mathrm{FB}^{\prime}$ is observed.

Holding levels are 11,000 ft and 14,000 ft for baseline and the LH procedure, respectively. A flight distance of $20 \mathrm{~nm}$ and $15 \mathrm{~nm}$ are found with an average of $155 \mathrm{~kg}$ (range 117-194 kg) and $112 \mathrm{~kg}$ (range $94-130 \mathrm{~kg}$ ) fuel burned for baseline and the LH procedure. From a level flights perspective, the average fuel saving is calculated to be $43 \mathrm{~kg}$ for the $\mathrm{LH}$ procedure. Assuming the same flight distance flown, a 3.6\% fuel saving is observed in favour of an LH. It should be highlighted that the significant fuel gain is provided by keeping aircraft higher $(14,000 \mathrm{ft})$ in the proposed procedure than conventional.

Even though there have been studies indicating lower performance of the BADA for fuel consumption estimation in terminal control area operations compared to cruise operation ${ }^{(45)}$, in the following paragraph, fuel consumption is found by using the BADA model ${ }^{(46)}$. In this regard, fuel consumption in delay conditions (i.e. the holding and sequencing legs) are evaluated considering the following parameters: aircraft gross mass, pressure altitude, time and Mach speed. In addition to these parameters, FPA is also taken into consideration for the descent segment. Table 3 indicates the constraints regarding BADA to calculate the FF for conventional holding and LH procedures. Moreover, aircraft mean mass, Mach speed, FPA and time duration for descent are presented for each $1,000 \mathrm{ft}$.

According to BADA, the fuel consumption is $39.3 \mathrm{~kg}$ and $59.7 \mathrm{~kg}$ in descent segment, and $136.8 \mathrm{~kg}$ and $98.9 \mathrm{~kg}$ in the level flight segments are found for conventional and $\mathrm{LH}$, respectively.

As presented in Table 4, regarding the comparison between baseline and LH procedures, the BADA model results are consistent with those using actual flight data. In both methods, the descent fuel consumption of LH is found to be $51.7 \%$ and $16.4 \%$ higher, compared to baseline, for BADA and actual flight data cases, respectively. Furthermore, for both methods, the fuel consumption at delay for the sequencing leg is found to be $27.8 \%$ lower than that for baseline. Considering that the BADA model tends to have higher performance for level flight, the same ratio of decrease in fuel consumption seems to be plausible. Lastly, the BADA model result concludes that switching the baseline procedure with $\mathrm{LH}$ yields a $10 \%(=17.6 \mathrm{~kg})$ fuel saving, whereas it is $16 \%(34.1 \mathrm{~kg})$ when using actual flight data. 
Table 3

BADA constraints to evaluate the FB' for both conventional holding and LH in the descent segment

$\begin{array}{lcccccccc}\text { Altitude (ft) } & \begin{array}{c}\text { Mean mass (tonne) } \\ \text { Conventional } \\ \text { Holding }\end{array} & \mathbf{L H} & \begin{array}{c}\text { Mach } \\ \text { Conventional } \\ \text { Holding }\end{array} & \mathbf{L H} & \begin{array}{c}\text { FPA } \mathbf{(}^{\circ} \text { ) } \\ \text { Conventional } \\ \text { Holding }\end{array} & \text { LH } & \begin{array}{c}\text { Time (second) } \\ \text { Conventional } \\ \text { Holding }\end{array} & \text { LH } \\ 22,000-21,000 & 57.3 & 57.5 & 0.57 & 0.55 & 3.98 & 3.14 & 25 & 32 \\ 21,000-20,000 & 57.6 & 57.6 & 0.58 & 0.54 & 3.98 & 3.14 & 24 & 33 \\ 20,000-19,000 & 57.6 & 57.6 & 0.57 & 0.53 & 3.98 & 3.14 & 24 & 33 \\ 19,000-18,000 & 57.4 & 57.7 & 0.56 & 0.53 & 3.98 & 3.14 & 25 & 33 \\ 18,000-17,000 & 57.5 & 57.7 & 0.55 & 0.52 & 3.98 & 3.14 & 26 & 34 \\ 17,000-16,000 & 57.4 & 57.7 & 0.53 & 0.52 & 3.98 & 3.14 & 26 & 34 \\ 16,000-15,000 & 57.7 & 57.7 & 0.53 & 0.51 & 3.98 & 3.14 & 26 & 34 \\ 15,000-14,000 & 57.7 & 57.7 & 0.52 & 0.49 & 3.98 & 3.14 & 27 & 35 \\ 14,000-13,000 & 57.9 & 57.6 & 0.50 & 0.49 & 3.98 & 3.43 & 28 & 32 \\ 13,000-12,000 & 57.7 & 57.5 & 0.47 & 0.46 & 3.98 & 3.43 & 28 & 34 \\ 12,000-11,000 & 57.6 & 57.9 & 0.46 & 0.46 & 3.98 & 3.43 & 29 & 34 \\ 11,000-10,000 & 57.9 & 57.7 & 0.44 & 0.45 & 1.89 & 3.43 & 65 & 35\end{array}$




\section{Table 4 \\ A summarized fuel consumption $(\mathrm{kg})$ results for baseline and $\mathrm{LH}$}

\begin{tabular}{ccccccc} 
& \multicolumn{3}{c}{ BASELINE } & & \multicolumn{2}{c}{ LH } \\
& \multicolumn{1}{c}{$\begin{array}{c}\text { Delay } \\
\text { Delay }\end{array}$} & \\
& Descent & (Holding) & Total & Descent & (Sequencing leg) & Total \\
$\begin{array}{c}\text { Based on an actual } \\
\text { flight data model }\end{array}$ & 56.08 & 155.20 & 211.28 & 65.26 & 111.90 & 177.16 \\
$\begin{array}{c}\text { Based on BADA } \\
\text { model }\end{array}$ & 39.31 & 136.81 & 176.12 & 59.66 & 98.86 & 158.52 \\
& & & & & &
\end{tabular}

\subsection{CONCLUSIONS}

In this study, LH based on PM is designed and proposed for Sivas Nuri Demirag Airport, Turkey, and comparative results are found in respect of conventional holding, in terms of air traffic management and fuel consumption.

Conventional holding stacks, commonly used for airborne delays, are available at the Sivas TMA. However, it is not possible to use them simultaneously due to overlapping buffer areas. Furthermore, as mentioned earlier, for conventional procedure, only one hold is considered. In the case of operational requirements, the number of holds could be increased. As the number of holds increases, the difference in fuel consumption between conventional holding and LH increases considerably. In addition, the recommended LH allows aircraft to fly at higher levels where lower engine thrust is needed, whereas it is the opposite case for conventional holding. Furthermore, LH enables aircraft to fly on predefined legs without any need for aircraft manoeuvring actions. Therefore, a controller has the chance to visualize a traffic situation easily and to be informed of the accurate traffic position. In addition, the RTS method is used to design and perform a validation of the LH procedure.

This study differs from earlier research in that it is the first LH study based on PM in which air traffic management and fuel consumption have been assessed at procedural TMA. Moreover, uniquely, 2,693 actual flights have been analysed focusing on descent flight between 22,000 ft and 10,000 ft. Two fuel consumption models, one for level flight at different altitudes and the other for the descent segment at different altitudes and FPAs are developed with the results being benchmarked.

The effects of altitude with a constant FPA (considered as 3.01-3.25 degrees) on FB' is analysed. For instance, the FB' is $8.2 \mathrm{~kg}$ between altitudes of $8,000-10,000 \mathrm{ft}$, whereas for altitudes of $10,000-12,000 \mathrm{ft}$, the FB' is found to be $7.3 \mathrm{~kg}$ with a reduction $11 \%$ for the descent segment. As a result, with a constant FPA, up to a $10 \%$ reduction can be observed up to $14,000 \mathrm{ft}$. Between altitudes of $14,000-22,000 \mathrm{ft}$, the FB' reduction is found to be less than $10 \%$.

From a level flights perspective, an average of $155 \mathrm{~kg}(117-194 \mathrm{~kg})$ and $112 \mathrm{~kg}(94-130 \mathrm{~kg})$ fuel burned for baseline and the LH procedure are found, respectively. The average fuel saving is calculated to be $43 \mathrm{~kg}$ for the LH procedure. Interestingly, if level flights at descent had not been considered, the proposed LH procedure would result in a fuel penalty of $9 \mathrm{~kg}$. This is because a shallower FPA is executed for the LH procedure where fuel consumption tends to be higher than it is for a steeper FPA.

The results of the analyses reveal that $16 \%$ total fuel savings can be achieved switching from baseline to the LH procedure for the current TMA. Furthermore, significantly higher 
fuel savings can also be obtained when the number of conventional holds increases. While these results are for a single TMA, they can also be generalized for other similar TMAs.

Furthermore, the results obtained by using the BADA model indicates that the $\mathrm{LH}$ is found to be $10 \%$ more advantageous compared to a conventional holding procedure, in terms of fuel consumption. Additionally, according to level flight, a $27.8 \%$ fuel reduction is found by implementing an LH.

As mentioned earlier, for a baseline procedure, only one hold is considered. In the case of operational requirements, the number of holds could be increased. As the number of holds increases, the difference of fuel consumption between the baseline and the LH will also increase considerably.

It should be noted that there are numerous local constraints, assumptions and different requirements to consider while designing the airspace. These local limitations are important to determine the flight procedures. In addition, it should be highlighted that the results of this research significantly depend on the design of the Sivas TMA and could not be applied to other TMAs. Therefore, in the future study, the LH under different local constraints should be evaluated.

\section{ACKNOWLEDGEMENTS}

The author ackowledge the EUROCONTROL which provided access to Base of Aircraft Data (BADA).

\section{REFERENCES}

1. Liang, M., Delahaye, D. and Marechal, M. "Integrated sequencing and merging aircraft to parallel runways with automated conflict resolution and advanced avionics capabilities", Transportation Research Part C: Emerging Technologies, 2017, 85, 268-291.

2. Zuniga, C.A., Piera, M.A., Ruiz, S. and Del Pozo, I. "A CD\&CR causal model based on path shortening/path stretching techniques", Transportation Research Part C, 2013, 33, 238-256.

3. ICAO, Doc 9426-Air Traffic Services Planning Manual, 1984, 1st ed., International Civil Aviation Organization, Montreal, Canada.

4. Delgado, L., Prats, X. and Sridhar, B. "Cruise speed reduction for ground delay programs: A case study for San Francisco International Airport arrivals", Transportation Research Part C, 2013, 36, 83-96.

5. Carlier, S., De Lépinay, I., Husatche, J.-C. and Jelinek, F. "Environmental impact of air traffic flow management delays", 2007, In: 7th USA/Europe Air Traffic Management Research and Development Seminar (ATM2007), Barcelona, Spain.

6. ICAO, DOC 8168, "Procedures for Air Navigation Services Aircraft Operations (PANSOPS)", 2006, 5th ed., Chapter II, International Civil Aviation Organization, Montreal, Canada.

7. ICAO, Doc 4444, "Procedures for Air Navigation Services Air Traffic Management", 2007, 15th ed., International Civil Aviation Organization, Montreal, Canada.

8. Gunther, T. and Fricke, H. "Potential of speed control on flight efficiency", in Proc. 2nd ICRAT (1), Belgrade, Serbia, 2006, 197-201.

9. Delgado, L. and Prats, X. "An en-route speed reduction concept for absorbing air traffic flow management delays", 2012, J Aircr, 49 (1), 214-224.

10. Prats, X. and Hansen, M. "Green delay programs", Ninth USA/Europe Air Traffic Management Research and Development Seminar (ATM2011), 2011, Berlin, Germany.

11. Delgado, L. and Prats, X. "Simulation of airborne ATFM delay and delay recovery by cruise speed reduction", 2011, In: Eurocontrol (Ed.), 1st SESAR Innovation days, Toulouse, France, pp. 1-7.

12. Delgado, L. and Prats, X. "Effect of wind on operating-cost-based cruise speed reduction for delay absorption", IEEE Transactions on Intelligent Transportation Systems, 2013, 14 (2), 918-927. 
13. Delgado, L. and Prats, X. "Operating cost-based cruise speed reduction for ground delay programs: Effect of scope length", Transportation Research Part C, 2014, 48, 437-452.

14. $\mathrm{XU}, \mathrm{Y}$. and Prats, $\mathrm{X}$. "Including Linear holding in air traffic flow management for flexible delay handling", $J$ Air Transportation, 2017, 25 (4), 123-137.

15. Slveling, G. and Clarke, J.-P, "Scheduling of airport runway operations using stochastic branch and bound methods", Transportation Research Part C: Emerging Technology, 2014, 45, 119-137.

16. Samá M., D'ariano, A., D'ariano, P. and Pacciarelli, D. "Optimal aircraft scheduling and routing at a terminal control area during disturbances", Transportation Research Part C, 2014, 47, $61-85$.

17. Samá M., D'ariano, A., D'ariano, P. and Pacciarelli, D. “Air traffic optimization models for aircraft delay and travel time minimization in terminal control areas", Public Transportation, 2015, 7(3), 321-337.

18. Akturk, S.M., AtAmturk A. and Gurel S. "Aircraft Rescheduling with Cruise Speed Control", Operations Research, 2014, 62 (4), 829-845.

19. https://skybrary.aero/bookshelf/books/2927.pdf, Accessed: 11.06.2018

20. Sahin, O., Usanmaz O. and Turgut, E.T. "An assessment of flight efficiency based on the point merge system at metroplex airports", Aircr Engineering and Aerospace Technology, 2018, 90 (1), $1-10$

21. Favennec, B., Vergne, F. and Zeghal, K. "Point merge integration of arrival flows enabling extensive RNAV application and CDA - operational services and environment definition", 2010, Eurocontrol Experimental Centre, Version 2.0.

22. http://www.eurocontrol.int/services/point-merge-concept. Accessed: March. 21, 2018.

23. Hong, Y., Chol, B., Lee, K. and Kim, Y. "Dynamic Robust Sequencing and Scheduling Under Uncertainty for the Point Merge System in Terminal Airspace", IEEE Transactions On Intelligent Transportation Systems, 2018, 19 (9), 2933-2943.

24. Sahin, O. and Usanmaz, O. "A new standard instrument arrival: The point merge system", Aircr Engineering and Aerospace Technology, 2013, 85 (2), pp. 136-143.

25. Ivanescu, D., Shaw, C., Tamvaclis, C. and Kettunen, T. "Models of air traffic merging techniques: Evaluating performance of point merge", 9th AIAA Aviation Technology, Integration, and Operations Conference (ATIO), Hilton Head, SC, USA, 2009.

26. Boursier, L., Favennec, B., Hoffman, E., Trzmiel, A., Vergne, F. and Zeghal, K. "Merging arrival flows without heading instructions", The 7th USA/Europe Air Traffic Management R\&D Seminar, Barcelona, Spain, 2007.

27. Barker, D.R., Haltli, B.M., Laqui, C., Macwilliams, P. and Mckee, K.L. "Assessment of terminal RNAV mixed equipage”, The 23rd IEEE Digital Avionics Systems Conference, Salt Lake City, UT, USA, 2004.

28. Klein, K.A., Sprong, K.R., Haltli, B.M., Becher, T.A. and Dearmon, J.S. "Evaluating operational benefits of terminal RNAV: Las Vegas case study", The 23rd IEEE Digital Avionics Systems Conference, Salt Lake City, UT, USA, 2004.

29. Laqui, C., Barker, D.R., Deamon, J.S. and Fawell, D.W. "Terminal RNAV: analyses of Las Vegas McCarran International Airport Terminal RNAV operations", The 22nd IEEE Digital Avionics Systems Conference, Indianapolis, IN, USA, 2003.

30. Cao, Y., Kotegawa, T., Sun, D., Delaurentis, D. and Post, J. "Evaluation of CDA as a standard terminal airspace operation", Ninth USA/Europe Air Traffic Management Research and Development Seminar, Berlin, Germany, 2011.

31. KHARDI, S. "Aircraft shortest and fastest continuous descent approach development", $J$ Aircr, 2012, 49 (6), 1931-1938.

32. ICAO DOC. 9931, "Continuous descent operations (CDO) manual", 2010, 1st Ed., International Civil Aviation Organization, Montreal, Canada.

33. https://www.eurocontrol.int/publication/real-time-simulation-dublin-tma (Accessed: 30.04 . 2019)

34. General Directorate of State Airports Authority, "Aeronautical Information Publication", 2019. http://ans.dhmi.gov.tr/ANSLogin.aspx?mn=41. (Accessed: 06.06.2019)

35. Usanmaz, O. and Turgut, E.T. "Wind effect analysis on instrument flight procedures using a Turkish wind model”, J Aircr, 2012, 49 (6), 2023-2032. 
36. EUROCONTROL, "Point merge integration of arrival flows enabling extensive RNAV application and continuous descent- operational services and environment definition", 2010.

37. Hong, Y., Lee, S., Lee, K. and Kim, Y. "Optimal Scheduling Algorithm for Air Traffic Point Merge System Using MILP", Advances in Aerospace Guidance, Navigation and Control, The fourth CEAS Specialist Conference on Guidance, Navigation and Control, 407-420, Warsaw, Poland, 2017.

38. LeE, S., Hong, Y. and KIM, Y. "Optimal scheduling algorithm in point merge system including holding pattern based on MILP", 7th European Conference for Aeronautics and Aerospace Sciences (EUCASS), Milan, Italy, 2017.

39. Hong Y., Choi, B., Lee, K. and Kim, Y. "Dynamic robust sequencing and scheduling under uncertainty for the point merge system in terminal airspace", IEEE Transactions on Intelligent Transportation Systems, 2017, 99, 1-11.

40. https://www.dhmi.gov.tr/Sayfalar/default.aspx, Accessed: 10.12.2018

41. Turgut, E.T. and Usanmaz, O. "Effect of climb angle on aircraft fuel consumption and nitrogen oxides emissions", J Aircr, 2018, 55 (6), 2392-2400. Doi:10.2514/1.C034265.

42. Padilla, C.E. Optimizing Jet Transport Efficiency: Performance, Operations and Economics, 1996, 1st ed., McGraw-Hill, New York, pp. 131-142.

43. Brentnall, A. and Cheng, R. "Some effects of aircraft arrival sequence algorithms", J Operational Research Soc, 2009, 60, 962-972.

44. Solveling G. and Clarke, J.P. "Scheduling of airport runway operations using stochastic branch and bound methods", Transportation Research Part C, 2014, 45, 119-137.

45. Senzig, D.A., Fleming, G.G. and Lovinelli, R.J. "Modeling of terminal-area airplane fuel consumption", J Aircraft, 2009, 46 (4), 1089-1093. https://doi.org/10.2514/1.42025

46. EUROCONTROL Experimental Centre, "User manual for the base of aircraft data (BADA)", 2017, Revision 3.14 . 